$$
\begin{aligned}
& u_{0}(x) \in C^{\alpha_{2}}[0,1], 0<\alpha_{2}<1 \\
& \text { 且. } h_{1}(0)=u_{0}(0), \\
& h_{1}(1)=u_{0}(1) .
\end{aligned}
$$

我们用 Schauder 不动点定理证明了如下的 局部存在定理.

定理 1 在 (A1) 与 (A2) 的假定下, 存在 $t_{1} \in\left(0, T\right.$ ], 使问题 $\left(\mathrm{N}_{\mathrm{p}}\right)$ 在 $Q_{t_{1}}$ 中 至少有一个弱解 $\{u(x, t), s(t)\}$, 它们满足:

$s(t) \in C^{2}\left[0, t_{1}\right]$ 且满足 $(4)$ 式, $u(x, t) \epsilon$ $C\left(\bar{Q}_{t_{1}}\right) \cap V_{2}^{1,0}\left(Q_{t_{1}}\right)$ 且满足 (2)与(3)式, 又对 任何 $t \in\left(0, t_{1}\right)$, 如下等式成立:

$$
\begin{gathered}
\int_{Q_{t}}\left\{-u(x, t) \varphi_{t}(x, t)+\left[u_{x}(x, t)\right.\right. \\
+g(u(s(t), t)) H(s(t)-x)] \\
\left.\quad \times \varphi_{x}(x, t)\right\} d x d t \\
-\int_{0}^{1} u_{0}(x) \varphi(x, 0) d x \quad \forall \varphi \in{\stackrel{\circ}{W_{2}^{1,1}}\left(Q_{t}\right)}^{\text {且 } \varphi(x, t)=0,}
\end{gathered}
$$$$
\text { 其中 } H(y)= \begin{cases}1 & \text { 当 } y>0 \\ 0 & \text { 当 } y \leqslant 0 .\end{cases}
$$

若进一步假定:

$$
\begin{array}{ll}
(\mathrm{A} 2)^{\prime} & h_{i}(t) \in C^{\alpha_{1}}[0, T], 1 / 2<a<1, \\
& i=1,2 . \\
& u_{0}(x) \in C^{\alpha_{2}}\left[0, s_{0}\right] \cap C^{\alpha_{2}}\left[s_{0}, 1\right],
\end{array}
$$

$$
\begin{aligned}
& 2<\alpha_{2}<3, \text { 且 } \\
& u_{0}\left(s_{0}^{+}\right)-u_{0}\left(s_{0}-\right), \\
& u_{0 x}\left(s_{0}+\right)-u_{0 x}\left(s_{0}-\right) \\
& \quad \quad-g\left(u_{0}\left(s_{0}\right)\right), \\
& h_{1}(0)-u_{0}(0), h_{2}(1)-u_{0}(1) .
\end{aligned}
$$

则有

定理 1' 在 (A1) 与 (A2)' 假定下, 定理 1 中的弱解也是经典解.

对于整体解的存在性问题, 如假定

$$
\text { (A1) } \begin{aligned}
& g(y) \in C^{1}(R), \text { 对一切 } y \in R, \text { 有 } \\
& |g(y)| \leqslant C(|y|+1) \text { 或 } \\
& y g(y) \geqslant 0,
\end{aligned}
$$

则有

定理 2 在 (A1) 与 (A2) 假定下, 问 题 ( $\mathrm{Np}$ ) 至少有一个弱解, 或者在整个区域 $Q_{T}$ 中, 或者在真子区域 $Q_{t_{1}}$ 中. 后者是解的 最大存在区域, 且有 $s\left(t_{1}\right)-0$ 或 $s\left(t_{1}\right)-1$.

类似于定理 $1^{\prime}$, 我们还有

定理 2' 在 (A1)' 与 (A2)' 假定下, 定理 2 中的弱解也是经典解.

\section{管志成}

(浙江大学数兴:系, 杭州 310027)

* 国家和断江省自然科学基金资助项目.

\title{
Connective Locales
}

本文引进 Connective Locales 的合适定 义, 证明了一个拓扑空间 $X$ 的开集格是 $\mathrm{Co}$ nnective Locale 当且仅当 $X$ 是 Connective 空 间,同时还讨论了 Connective Locale 的一些 性质. 这样, Locale 理论 ${ }^{[1]}$ 得到了进一步的 完善.

定义 1 Locale $A$ 被称为是. Connective Locale, 若对任意 Locale 映射 $t: A \rightarrow$ $\searrow_{0}, f^{*}(a)-1_{A}$ 或 $f^{*}(b)-1_{A}$.

定球 1 设 $A$ 是 Locale, 则下述条件 等价: (i) $A$ 不是 Connective;

(ii) 存在 Locale 映射 $f: A \rightarrow>_{b}$, 使得 $f^{*}(a) \neq 1_{A}$, 且 $f^{*}(b) \neq 1_{A}$;

(iii) 存在 $x, y \in A$, 使得 $x \vee y=1_{A}$, $x \wedge y-0_{A}, x, y \neq 1_{A}$;

(iv) 存在 $x \in A$, 使得 $x \gtrless x$.

定理 2 设 $f: A \rightarrow B$ 是 Locale 映射 且 $f^{*}(x)-1_{A} \Longleftrightarrow x-1_{\text {s. }}$ 那么若 $A$ 是 Connective, $B$ 亦然.

定理 3 若 Locale $A$ 包含一个稠子 Locale $A_{i}$ 是 Connective, 则 $A$ 也是 Conne- 
ctive.

推论 1 若 Locale $\boldsymbol{A}$ 有 Flat sublocale $A_{i}$ 是 Connective, 则 $A$ 也是 Connective.

定理 4 Spatial Locale $A$ 是 Connective 当且仅当拓扑空间 Spec $(A)$ 是连通 空间.

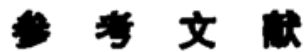

[1] Johnstone, P. T., Stone Spaces, Cambridge Univ. Press, Cambridge, 1982.

白仲林

(西北师范大学数学系, 兰州 730070)

\section{关于 $x+y_{2} \rightarrow x y+y$ 反应的速率常数的计算}

体系的过渡态是直线型的, 有效势能为

$$
U\left(r_{1}\right)=V\left(r_{1}\right)+\frac{l(l+1) \hbar^{2}}{2 I\left(r_{1}\right)},
$$

$l$ 为转动量子数, $M_{1}$ 和 $M_{2}$ 分别是 $\mathrm{y}$ 原子和 $\mathrm{x}$ 原子的质量, $r_{1}$ 和 $r_{2}$ 分别是 $\mathrm{y}$ 原子间及 $x$ 与较近的一个 $y$ 原子间的距离. 体系对于质 心的转动惯量是

$$
\begin{aligned}
I\left(r_{1}\right)= & \left\{2 M_{1} M_{2} r_{2}^{2}+\left(M_{1}+M_{2}\right) M_{1} r_{1}^{2}\right. \\
& \left.+2 r_{1} r_{2} M_{1} M_{2}\right\} /\left(2 M_{1}+M_{2}\right),
\end{aligned}
$$

由反应讨程中的键级守恒 ${ }^{[1]}, r_{2}$ 可表示为 $r_{1}$ 的函数. $V\left(r_{1}\right)$ 是两个 Lennard-Jones 势. 过渡态的条件是

$\left.\frac{d U}{d r_{1}}\right|_{R_{1}}=0$, 记 $V^{\prime}=\left.\frac{d V}{d r_{1}}\right|_{R_{1}} I^{\prime}-\left.\frac{d I}{d r_{1}}\right|_{R_{1}}$.

$R_{1}$ 是过渡时 $\mathrm{y}$ 原子间的距离, 即临界键长. 平均临界键长为

$$
\bar{R}_{1}-\frac{1}{Q} \int e^{-\frac{V\left(R_{1}\right)}{d T}} F\left(R_{1}\right) R_{1} d R_{1},
$$

式中的 $Q$ 为归一化因子, $F\left(R_{1}\right)$ 由下式确定:

$$
\begin{aligned}
F\left(R_{1}\right)= & \frac{1}{\hbar^{2}}\left[\frac{d^{2} V}{d R_{1}^{2}} \frac{2 I^{2}\left(R_{1}\right)}{I^{\prime}}\right. \\
& +\frac{4 V^{\prime} I\left(R_{1}\right)}{I^{\prime}} \frac{d I\left(R_{1}\right)}{d R_{1}} \\
& \left.-\frac{2 V^{\prime} I^{2}\left(R_{1}\right)}{I^{\prime 2}} \frac{d I^{\prime}}{d R_{1}}\right] .
\end{aligned}
$$

冻络反应坐标时, 过度态的配分函数为

$$
\begin{aligned}
Q^{ \pm}- & {\left[\frac{2 \pi\left(2 M_{1}+M_{2}\right)}{h^{2}} k T\right]^{3 / 2} \frac{8 \pi^{2} I\left(\bar{R}_{1}\right) k T}{h^{2}} } \\
& \prod_{i=1}^{3} \frac{e^{-\frac{A v i}{2 k T}}}{h^{-\frac{h p i}{k T}}}
\end{aligned}
$$

其中的振动频率由文献 [1] 中的方法求得. 反应物的配分函数为

$$
\begin{gathered}
Q=\left[\frac{4 \pi M_{1} k T}{h^{2}}\right]^{3 / 2}\left[\frac{2 \pi M_{2} k T}{h^{2}}\right]^{3 / 2} \\
\cdot \frac{8 \pi^{2} I^{\prime} k T}{2 h^{2}} \frac{e^{-\frac{h \nu^{\prime}}{2 k T}}}{1-e^{-\frac{h \nu^{\prime}}{k T}} Q_{E},}
\end{gathered}
$$

$I^{\prime}$ 是 $y_{2}$ 分子关于其质心的转动惯量, $v^{\prime}$ 是 $y_{2}$ 的振动频率. 电子的配分函数 $Q_{k}-2$. 反应速率常数为

$$
K=\frac{k T}{h} \frac{Q^{ \pm}}{Q} e^{-\frac{V\left(R_{1}\right)-V\left(r_{10}\right)}{k T}},
$$

$V\left(\bar{R}_{1}\right)$ 可认为是过渡态的基态平均能量. $V\left(r_{10}\right)$ 是反应物的基态能量. 由(5)式可得 计算活化能和指前因子的公式如下:

$$
\begin{gathered}
\Delta E=R T^{2} \frac{\partial \ln K}{\partial T}, \\
A=\frac{k T}{h} e^{2} e^{\frac{\Delta s}{R}}, \quad \Delta S-R \frac{\partial}{\partial T}\left[T \ln \frac{Q^{ \pm}}{Q}\right],
\end{gathered}
$$

利用以上理论讨论了下列三种反应: $\mathrm{F}+\mathrm{H}_{2} \longrightarrow \mathrm{FH}+\mathrm{H}, \mathrm{O}+\mathrm{H}_{2} \longrightarrow \mathrm{OH}+\mathrm{H}$ 和 $\mathrm{H}+\mathrm{D}_{2} \longrightarrow \mathrm{HD}+\mathrm{D}$, 计算了它们的平均 临界键长, 以确定过渡态的构型. 也计算了 反应活化能、指前因子和反应速率常数。所 用常数来自文城 $[2,3]$. 计算结果与实验值 符合得很好。

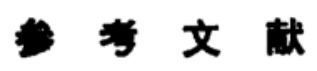

[1] Johnston, H.S. and Goldfinger, P., J. Chem. Phys., 37(1962), 700. 